\title{
Paley's Multiplier Method Does Not Accurately Predict Adult Height in Children with Bone Sarcoma
}

\author{
Magdalena Maria Gilg MD, Christine Wibmer MD, Dimosthenis Andreou MD, \\ Alexander Avian PhD, Petra Sovinz MD, Werner Maurer-Ertl MD, \\ Per-Ulf Tunn MD, Andreas Leithner MD
}

Received: 1 October 2013/ Accepted: 7 April 2014/Published online: 29 April 2014

(C) The Association of Bone and Joint Surgeons (B) 2014

\begin{abstract}
Background The majority of patients with osteosarcoma and Ewing's sarcoma are diagnosed before skeletal maturity. Paley's multiplier is used for height prediction in healthy children, and has been suggested as a method to make growth predictions for children with osteosarcoma and Ewing's sarcoma when considering limb salvage options. To our knowledge, no evaluation of this method in this particular patient group has been performed, but a
\end{abstract}

Each author certifies that he or she, or a member of their immediate family, has no commercial associations (eg, consultancies, stock ownership, equity interest, patent/licensing arrangements, etc) that might pose a conflict of interest in connection with the submitted article.

All ICMJE Conflict of Interest Forms for authors and Clinical Orthopaedics and Related Research editors and board members are on file with the publication and can be viewed on request.

Each author certifies that his or her institution approved the human protocol for this investigation, that all investigations were conducted in conformity with ethical principles of research, and that informed consent for participation in the study was obtained.

This work was performed at the Department of Orthopaedic Surgery, Graz, Austria; and the Department of Orthopaedic Oncology, HELIOS Klinikum Berlin-Buch, Berlin, Germany.

M. M. Gilg, C. Wibmer (凹), W. Maurer-Ertl, A. Leithner Department of Orthopaedic Surgery, Medical University of Graz, Auenbruggerplatz 5, 8036 Graz, Austria

e-mail: christine.wibmer@medunigraz.at

D. Andreou

Department of General Orthopaedics and Tumororthopaedics, University Hospital of Muenster, Münster, Germany

\section{A. Avian}

Institute for Medical Informatics, Statistics and Documentation,

Medical University of Graz, Graz, Austria temporary growth deficit has been observed in children undergoing chemotherapy.

Questions/purposes We asked whether (1) Paley's formula reliably predicts growth in children who received polychemotherapy; (2) chemotherapy impairs growth velocity; and (3) final adult height is impaired in these patients.

Methods Retrospectively, data for 94 patients with osteosarcoma and Ewing's sarcoma were retrieved from databases of two sarcoma centers. Onset before 14 years of age in girls and 16 years in boys and a minimum followup until 18 years were required (mean, 67 months; range, 31-124 months) criteria. Exclusion criteria were the intake of growth hormones or no chemotherapy. Thirty-three patients (35\%) fulfilled all inclusion criteria. Predicted adult heights were compared with actual adult height. The development of a growth deficit was evaluated for 23 children (without chemotherapy for recurrence) using ageand gender-specific standard deviation scores for height (WHO Z-scores).

Results Height prediction using Paley's method showed a high percentage of false predictions (outside $\pm 1 \mathrm{SD}, 70 \%$; outside $\pm 2 \mathrm{SD}, 61 \%$ ). On average, the mean total height of the patients was overestimated $(2.3 \mathrm{~cm})$. The median

\footnotetext{
P. Sovinz

Division of Paediatric Haemato-Oncology, Department of Paediatrics, Medical University of Graz, Graz, Austria

P.-U. Tunn

Department of Orthopaedic Oncology, HELIOS Klinikum

Berlin-Buch, Berlin, Germany
} 
absolute error of prediction was $5.0 \mathrm{~cm}$ (range, -17 to 8 ). Patients with osteosarcoma and Ewing's sarcoma showed a significant growth impairment during polychemotherapy. A catchup phase in growth before skeletal maturity was observed in patients with osteosarcoma but not with Ewing's sarcoma.

Conclusions Owing to its lack of reliability in this patient group, methods other than Paley's should be evaluated to predict adult height. Although limited by a small number of patients, our study results indicate a decreased adult height in patients with bone sarcoma after chemotherapy.

Level of Evidence Level III, therapeutic study. See the Instructions for Authors for complete description of levels of evidence.

\section{Introduction}

Osteosarcoma and Ewing's sarcoma are the two most common malignant bone tumors in children and adolescents, accounting for $5 \%$ of all malignant tumors in these age groups, with a peak incidence between 10 and 14 years of age $[2,25]$. Treatment follows standardized study protocols comprising neoadjuvant polychemotherapy and surgery with wide resection margins followed by adjuvant polychemotherapy. Patients with Ewing's sarcoma also may receive radiation therapy $[2,28]$. After implementation of chemotherapy, the 5-year-survival rate increased from $15 \%$ to as much as $50 \%$ to $70 \%$ for patients with osteosarcoma and from 10\% to as much as $50 \%$ to $75 \%$ for patients with Ewing's sarcoma [5]. Limb salvage often can be performed and reconstruction possibilities include expandable endoprostheses among other options [1, 22]. Early during the experience with expandable endoprostheses, multiple operations were necessary to limit limb length discrepancy when the patients reached skeletal maturity [18]. The first expandable prostheses were implanted in 1976 and each lengthening required a minor surgical intervention to achieve expansion [1, 22, 33]. Technologic developments now allow noninvasive lengthening of the endoprostheses $[18,20]$.

To select the patients who will benefit from an expandable prosthesis, a reliable prediction of the expected limb length discrepancy is needed. A multiplier method for prediction of total body height was introduced by Paley et al. [24] for which no radiographs are necessary. It is based on 52 databases of healthy children containing growth data collected from 1951 until 2003. This is in contrast to previously presented methods like bone age determination according to Greulich and Pyle [15], the Tanner-Whitehouse method [35], the Roche-Wainer-Thissen method [31], or Paley's multiplier method for the lower limb [23]. Comparison between the Greulich and Pyle and Paley's lower limb multiplier methods [32] was conducted although no explicit evaluation of the total height multiplier was reported. It has been suggested that Paley's multiplier for height prediction can be used in children being treated for bone sarcoma, although it has not been prospectively tested in children who are treated with polychemotherapy $[4,24]$.

Aberrant growth resulting from chemotherapy was first observed in children who had received intensive chemotherapy and cranial irradiation for acute lymphatic leukemia [16, 17, 39]. These patients had a decline in growth velocity while receiving intensive chemotherapy. After cessation of antineoplastic treatment, children showed catchup growth [39]. A similar decrease in growth velocity was seen in patients with osteosarcoma and Ewing's sarcoma during and 1 year after chemotherapy $[3,14]$. Additionally, it was clinically suspected that patients with osteosarcoma and Ewing' sarcoma never catch up with their peers until skeletal maturity [14]. This observation has not been validated in clinical studies $[8$, 14]. Murine studies have shown a direct inhibitive effect of some of the administered antineoplastic agents on the growth plate [29, 30, 36-38, 41, 42].

The aims of our study were to determine whether (1) Paley's formula reliably predicts growth in children who received polychemotherapy for bone sarcoma, (2) chemotherapy impairs growth velocity, and (3) final adult height is impaired in patients with osteosarcoma and Ewing's sarcoma.

\section{Patients and Methods}

To identify growth deficits and validate Paley's multiplier method in patients with osteosarcoma and Ewing's sarcoma, a retrospective database review was conducted.

We identified 94 patients in the tumor databases of our two departments, younger than 14 years for girls and 16 years for boys, with the initial diagnosis of osteosarcoma or Ewing's sarcoma between 1990 and 2009. Followup data for height had to be available until the patient was 18 years old, which was chosen as the endpoint of the analysis in concordance with the (age) specifications of the multiplier method and the WHO growth reference data [10, 11]. Fortyfour patients had to be excluded because of incomplete clinical data (height at initial diagnosis, height at maturity) or contradicting height documentation. Further exclusion criteria were the intake of growth hormones (one patient), lack of chemotherapy (three patients), epiphyseodesis (no patients), cranial irradiation (no patients), and followup at external institutions (13 patients). The presence of metastases at the time of initial diagnosis was not an exclusion 
Table 1. Patient characteristics

\begin{tabular}{llll}
\hline Characteristic & Ewing's sarcoma N =17 & Osteosarcoma N = 16 & Total N $=33$ \\
\hline Male:female & $15: 2$ & $12: 4$ & $27: 6$ \\
Mean age at diagnosis (range) (years) & $12.1 \pm 2.5(8-16)$ & $12.8 \pm 1.8(9-16)$ & $12.5 \pm 2.2(8-16)$ \\
Median duration of chemotherapy (range) (months) & $10 \pm 2.1(5-12)$ & $8 \pm 1.5(4-10)$ & $9 \pm 2.2(4-12)$ \\
Mean time of followup (range) (months) & $71 \pm 29(31-124)$ & $61 \pm 20(3-97)$ & $66.1 \pm 25(3-124)$ \\
Mean height (at 18 years of age) $(\mathrm{cm})$ & $175.2 \pm 9.4(160-193)$ & $174.3 \pm 8.2(159-187)$ & $175.2 \pm 8.7(159-193)$ \\
\hline
\end{tabular}

Table 2. Growth prediction

\begin{tabular}{llll}
\hline Growth prediction & $1 \mathrm{SD}$ & $2 \mathrm{SD}$ & $3 \mathrm{SD}$ \\
\hline Correct & 10 & 13 & 24 \\
& $30 \%$ & $39 \%$ & $73 \%$ \\
False & 23 & 20 & 9 \\
& $70 \%$ & $61 \%$ & $27 \%$ \\
Overestimation & 18 & 15 & 7 \\
Underestimation & 5 & 5 & 2 \\
Total & 33 & 33 & 33 \\
\hline
\end{tabular}

criterion as it is postulated that a majority of patients with bone sarcoma have nondetectable metastatic disease (micrometastasis) [28, 34]. All sarcoma locations were included in the study. Thirty-three patients, 16 with osteosarcoma and 17 with Ewing's sarcoma (male-to-femaleratio, 4.5:1), remained as the subjects of this study (Table 1). Followup ranged between 31 and 124 months (mean, 66 months). Mean age at diagnosis was 12.8 years for patients with osteosarcoma (range, 9-16 years) and 12.1 years (range, 8-15 years) for patients with Ewing's sarcoma. As of August 2012, 27 patients were disease-free at followup, five patients had died of disease, and one patient was alive with disease.

The expected adult height for these patients was calculated with Paley's multiplier method and compared with their actual adult height. For analysis of growth impairment, Z-scores (age- and gender-related SD scores for height) according to WHO child growth standards were calculated at the beginning and end of chemotherapy and when the patient was 18 years old $[10,11]$. For this calculation 10 patients who received chemotherapy for relapse were excluded because end points were not comparable to those who had standard chemotherapy.

All children with osteosarcoma were treated according to the Cooperative Osteosarcoma Study group (COSS) protocols active at the time of diagnosis (Appendix 1) [6, 7, 40]. Patients with Ewing's sarcoma were treated with the EUROpean Ewing tumour Working Initiative of National Groups (EURO-EWING) 99 or European Intergroup Cooperative Ewing's Sarcoma Study (EICESS) 92 protocols $[18,20,25]$. The median duration of chemotherapy was
8 months (range, 4-10 months) in the osteosarcoma group and 10 months (range, 5-12 months) in the Ewing's sarcoma group (Appendix 2) [19, 21, 26]. Initial body heights were retrieved from patients' records. Followup regarding height was available through regular measurements at the pediatric outpatient clinics. Assessment of height was done before midday to minimize the influence of the physiologic circadian variability. This assessment was done by trained nurses using an electronic device. Height values were recorded with one digit after the decimal point in the patients' records, the medical reports, local databases, and protocols of chemotherapy. If adult height was not documented, patients were contacted by phone to obtain their adult height (necessary for four [12\%] patients).

Statistical analysis was conducted with SPSS $^{\circledR} 20$ $\left(\mathrm{IBM}^{\circledR}\right.$, Armonk, NY, USA). The values of continuous variables are presented as mean and SD. Categorical variables are described as absolute and relative frequencies. Predicted height was calculated with appropriate multipliers and the height difference, adult height minus predicted height, was calculated. To determine growth impairment age- and gender specific standard deviation scores were calculated as follows: Z-scores $(Z$ start $=Z$-score at the beginning of chemotherapy, $Z$ end $=Z$-score at the end of chemotherapy, Z $18=\mathrm{Z}$-score at the age of 18 years) were obtained according to WHO growth standards [10, 11].

If the assumption of normal distributed data was met, continuous variables were analyzed using a dependent t-test. Otherwise, nonparametric methods (Wilcoxon signed ranks test) were used. A p value less than 0.05 was considered significant. Approval by the institutional review board was obtained (EK number 23-440).

\section{Results}

A discrepancy between actual and predicted height was observed after applying Paley's formula in our dataset. The estimated adult height was overestimated in the majority $(76 \%)$ of patients in absolute terms. With a SD of 1 , two of three children had a false height prediction (five too low, 18 too high). Within 2 SD the percentage of false predictions decreased to approximately $60 \%$ of the children (five 


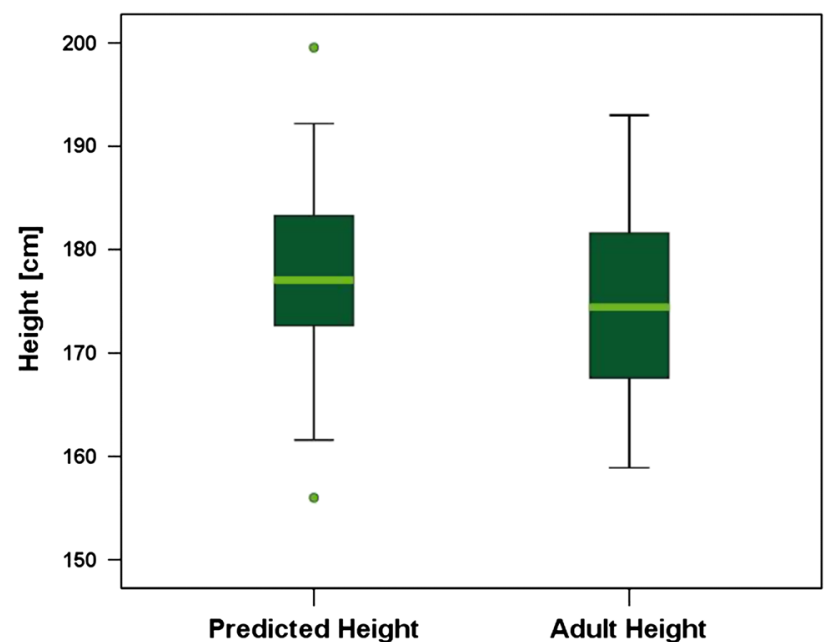

Fig. 1 The predicted body height is greater than the actual adult height.

Table 3. Z-scores for patients with Ewing' s sarcoma

\begin{tabular}{|c|c|c|c|c|c|c|}
\hline $\begin{array}{l}\text { Patient } \\
\text { number }\end{array}$ & Sex & $\mathrm{Z}$ start & $\mathrm{Z}$ end & Z 18 & $\begin{array}{l}Z \text { start to } \\
Z \text { end }\end{array}$ & $\begin{array}{l}Z \text { start to } \\
Z 18\end{array}$ \\
\hline 1 & $\mathrm{M}$ & +0.71 & -0.88 & -0.63 & -1.59 & -1.34 \\
\hline 2 & $\mathrm{~F}$ & +0.87 & +0.19 & +0.17 & -0.68 & -0.70 \\
\hline 3 & $\mathrm{M}$ & +0.11 & -0.46 & +0.11 & -0.57 & 0.00 \\
\hline 4 & $\mathrm{~F}$ & -0.16 & -0.73 & -0.47 & -0.57 & -0.31 \\
\hline 5 & $\mathrm{M}$ & +2.96 & +2.51 & +1.85 & -0.45 & -1.11 \\
\hline 6 & $\mathrm{M}$ & +0.53 & +0.12 & -0.27 & -0.41 & -0.80 \\
\hline 7 & $\mathrm{M}$ & +1.31 & +0.92 & +0.89 & -0.39 & -0.42 \\
\hline 8 & $\mathrm{M}$ & -0.10 & -0.40 & -0.44 & -0.30 & -0.34 \\
\hline 9 & $\mathrm{M}$ & +0.43 & +0.20 & +0.25 & -0.23 & -0.18 \\
\hline 10 & M & +0.88 & +0.67 & +0.76 & -0.21 & -0.12 \\
\hline 11 & $\mathrm{M}$ & +2.04 & +1.92 & +2.11 & -0.12 & +0.07 \\
\hline 12 & $\mathrm{M}$ & -0.85 & -0.77 & +0.25 & +0.08 & +1.10 \\
\hline 13 & $\mathrm{M}$ & -0.45 & -0.15 & -1.40 & +0.30 & -0.95 \\
\hline
\end{tabular}

too low, 15 too high). Within $3 \mathrm{SD}$, one of four patients had a false prediction (two too low, seven too high) (Table 2). The median absolute error of prediction was $5.0 \mathrm{~cm}$. Median height difference was $-5.0 \mathrm{~cm}$ (range, -17 to $-0.6 \mathrm{~cm}$ ) in children whose height was overestimated and $5.4 \mathrm{~cm}$ (range, $0.5-8 \mathrm{~cm}$ ) in those whose height was underestimated. On average the adult height of the study population was overestimated: the mean predicted height was $2.3 \mathrm{~cm}$ greater than the adult height $(\mathrm{p}=0.013)$ (Fig. 1).

The mean height at diagnosis of all patients was greater than in the reference population $(\mathrm{Z}$ start osteosarcoma $=$ +0.55 [Table 3], $Z$ start Ewing's sarcoma $=+0.64$
Table 4. Z-scores for patients with osteosarcoma

\begin{tabular}{lllllll}
\hline $\begin{array}{l}\text { Patient } \\
\text { number }\end{array}$ & Sex & Z start & Z end & Z 18 & $\begin{array}{l}\text { Z start to } \\
\text { Z end }\end{array}$ & $\begin{array}{l}\text { Z start to } \\
\text { Z 18 }\end{array}$ \\
\hline 1 & $\mathrm{M}$ & -1.03 & -1.63 & -1.48 & -0.60 & -0.45 \\
2 & $\mathrm{M}$ & -1.11 & -1.50 & -1.21 & -0.39 & -0.10 \\
3 & $\mathrm{M}$ & -1.59 & -1.33 & -0.68 & +0.26 & +0.91 \\
4 & $\mathrm{M}$ & +0.14 & -0.36 & +0.37 & -0.50 & +0.23 \\
5 & $\mathrm{M}$ & +0.43 & +0.29 & +0.48 & -0.14 & +0.05 \\
6 & $\mathrm{~F}$ & +1.54 & +1.11 & +0.74 & -0.43 & -0.80 \\
7 & $\mathrm{M}$ & +0.84 & +0.24 & +0.91 & -0.60 & +0.07 \\
8 & $\mathrm{M}$ & +2.02 & +1.73 & +1.38 & -0.29 & -0.64 \\
9 & $\mathrm{~F}$ & +2.03 & +1.87 & +1.81 & -0.16 & -0.22 \\
10 & $\mathrm{~F}$ & +2.24 & +1.97 & +2.23 & -0.27 & -0.01 \\
\hline
\end{tabular}

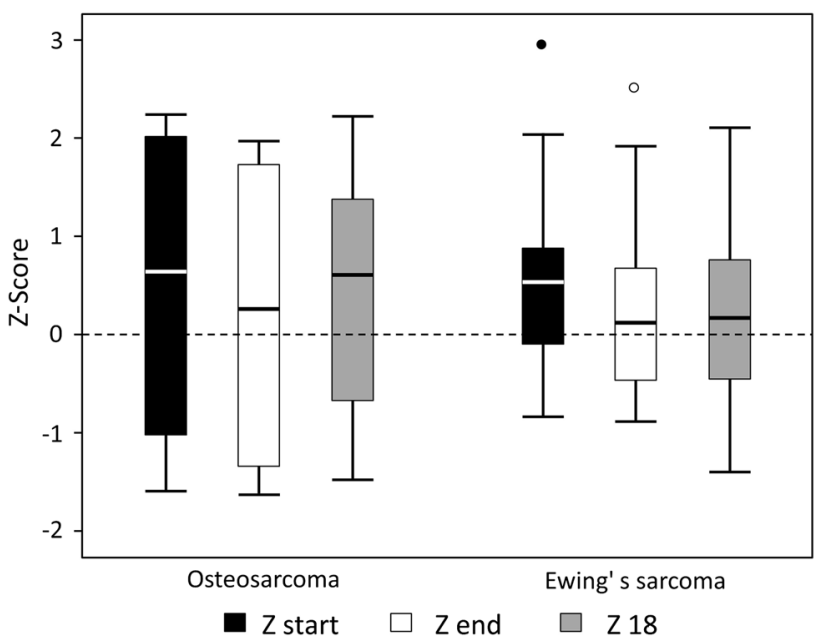

Fig. 2 Z-scores for the beginning of chemotherapy ( $Z$ start), the end of chemotherapy ( $\mathrm{Z}$ end) and at the age of 18 years $(\mathrm{Z} 18)$ for patients with osteosarcoma and with Ewing's sarcoma are shown.

[Table 4]). A growth deficit at the end of chemotherapy was seen in all patients $(\mathrm{Z}$ end osteosarcoma $=0.24$, decrease $p=0.004, Z$ end Ewing's sarcoma $=0.24$, decrease $p=0.008$ ) relative to WHO growth charts.

A catchup in growth until adulthood was seen for patients with osteosarcoma only: $\mathrm{Z}$ start was not significantly different from Z $18(\mathrm{Z}$ start $=0.55, \mathrm{Z} 18=0.46$, $\mathrm{p}=0.546$ ). For patients with Ewing's sarcoma, a permanent growth deficit was observed $(\mathrm{Z}$ start $=0.64, \mathrm{Z}$ $18=0.34, \mathrm{p}=0.043$ ) (Fig. 2).

\section{Discussion}

Paley's multiplier method for height prediction was designed for healthy children with limb length discrepancies and was not evaluated for patients with bone sarcoma 
who received polychemotherapy. A decrease in growth velocity while receiving intensive chemotherapy has been observed in patients with bone sarcoma. Interference of antineoplastic agents with growth has been observed in children with acute lymphatic leukemia [16, 17, 39]. In addition, a direct negative effect of antineoplastic agents on the growth plate was detected in murine studies [29, 30, 36-38, 41, 42]. Therefore, we analyzed (1) Paley's formula for height prediction of children with bone sarcoma, (2) the effect of polychemotherapy on growth, and (3) a possible catchup growth for patients with osteosarcoma and those with Ewing's sarcoma.

We acknowledge some limitations of this study. First, from 94 patients initially, only 33 patients remained subjects of the study owing to specific inclusion criteria and the retrospective study design. The age restriction and need for complete followup until adulthood further contributed to the small patient sample. Second, the male-to-female ratio in our study population was $4.5: 1$ which differs from the normal sex distribution of patients with these malignancies (1.08:1.0) [25]. As we have a small study collective, this abnormal ratio might be accidental. Even though Paley's multiplier values and Z-scores are adjusted to gender one must be careful when generalizing our results to both genders [24]. Third, as a result of risk stratification in the study protocols and dose reduction resulting from adverse effects, no subgroup analysis concerning the effect of chemotherapy intensity on adult height could be performed in this small study collective. Fourth, two patients $(6 \%)$, one female, 14 years old with a height at diagnosis of $175 \mathrm{~cm}$, and one male, 15 years old with a height at diagnosis of $182 \mathrm{~cm}$, did not show any growth, although additional growth was expected according to Paley's method. Whether growth plates were already closed in these two patients or this was an effect of chemotherapy cannot be distinguished in the retrospective study design. Fifth, the retrospective study design involves the possibility of documentation errors, because height measurements were recorded manually in the outpatient notes. In addition, adult height was obtained by contacting patients by phone (four patients [12\%]), and these measurements may have been less accurate.

Regarding the accuracy achieved with the multiplier method, we see a median absolute error of prediction of $\pm 5 \mathrm{~cm}$ in total body height in our collective. This has to be taken into account when using Paley's multiplier method in this particular patient group.

The observed decrease in growth velocity at the end of chemotherapy in our study population is comparable to published data concerning patients with acute lymphatic leukemia [9, 16, 17, 39] and bone sarcoma [3, 8, 14]. At skeletal maturity the growth deficit was still detectable in our data set, albeit more pronounced in patients with Ewing's sarcoma than in patients with osteosarcoma. It is somewhat challenging to compare our data with the data of others, as the number of clinical studies with complete followup through adulthood is limited [8, 14]. Cool et al. [8] observed catchup growth until skeletal maturity in a collective of 16 pediatric patients with osteosarcoma. Glasser et al. [14] initially said, according to results of a pilot study, that after chemotherapy, children have lost 1 $\mathrm{SD}$ in adult height, corresponding to $5.6 \mathrm{~cm}$ in girls and $6.6 \mathrm{~cm}$ in boys. They could not confirm this, however, in the subsequent study population of 122 patients with osteosarcoma and Ewing's sarcoma [14]. In their group, $80 \%$ of patients showed a height difference less than 1 SD between initial diagnosis and skeletal maturity [14]. Based on these results, they concluded that growth velocity is affected while patients undergo chemotherapy; however, attained heights at maturity were within population norms [14]. Consistent with Glasser et al. [14], the height deficit in our data set was less than $1 \mathrm{SD}$ and the absolute height deficit in a moderate range (less than $3 \mathrm{~cm})$.

Regarding the influence of polychemotherapy on final adult height, and because we observed greater growth retardation in patients with Ewing's sarcoma compared with those with osteosarcoma, we analyzed differences between the two entities such as age at initial diagnosis, duration of chemotherapy, and the administered antineoplastic agents. The age of patients at onset of Ewing's sarcoma is usually 1 to 3 years earlier than for osteosarcoma [25]. Therefore, the remaining growth period is expected to be longer for patients with Ewing's sarcoma. In our data set, the difference was only 7 months (average onset at 12.8 years in patients with osteosarcoma versus 12.1 years in patients with Ewing's sarcoma), so this factor does not adequately explain the growth deficit. Average treatment duration is longer for patients with Ewing's sarcoma (7.6 months for osteosarcoma versus 10.0 months for Ewing's sarcoma) leading to increased cumulative dosages of chemotherapy agents. The influence of several antineoplastic agents on the growing bone has been analyzed in murine studies [29, 30, 36-38, 41, 42]. All antineoplastic agents, except for methotrexate, used for osteosarcoma and Ewing's sarcoma seem to affect the growth plate, in particular the proliferative zone. Although some studies did not detect an effect of methotrexate on the growth plate, some authors agree that it affects bone quality and can cause osteoporosis [12, 13, 27]. It is not clear whether the observations found in the murine studies are transferable to humans [29]. Furthermore, there are no 
data in the literature, to our knowledge, regarding whether the higher growth retardation in patients with Ewing's sarcoma is the consequence of a single agent or an accumulation of agents.

Based on our results, Paley's multiplier method should not be the sole tool relied on when predicting adult height in pediatric patients being treated for bone sarcoma. Accurate information on adult height might be useful in making the choice of limb salvage reconstruction options such as the use of an expandable prosthesis or other reconstruction alternatives. Being able to predict adult height would allow the surgeon to choose an implant with enough expansion ability to be able to equalize limb length at skeletal maturity. Unfortunately the Paley multiplier method appears to be an inaccurate method of predicting adult height in patients with bone sarcoma because of its high percentage of imprecise predictions for patients with osteosarcoma and those with Ewing's sarcoma, with overestimation of adult height being the case in the majority of patients. Currently no validated new tool for this particular patient group has been developed. To evaluate other growth prediction methods, additional studies need to be performed. Regarding long-term growth development in patients with bone sarcoma, we observed lasting growth retardation in patients with osteosarcoma and those with Ewing's sarcoma. For explicit answers concerning primary causes for the height impairment, further studies are needed.

\section{Appendix 1: Chemotherapy in osteosarcoma}

\begin{tabular}{|c|c|c|c|c|c|}
\hline \multirow[t]{2}{*}{ Protocol } & \multicolumn{3}{|l|}{ Neoadjuvant } & \multicolumn{2}{|l|}{ Adjuvant } \\
\hline & LR & HR 1 & HR 2 & LR & HR $1 / 2$ \\
\hline \multirow[t]{4}{*}{ COSS-86 } & Methotrexate & Methotrexate & Methotrexate & Methotrexate & Methotrexate \\
\hline & Adriamycin & Adriamycin & Adriamycin & Adriamycin & Adriamycin \\
\hline & Cisplatin & Cisplatin* & Cisplatin* & Cisplatin & Cisplatin \\
\hline & & Ifosfamide* & Ifosfamide* & & Ifosfamide \\
\hline \multirow[t]{2}{*}{ Protocol } & \multirow[t]{2}{*}{ Neoadjuvant } & \multicolumn{4}{|l|}{ Adjuvant } \\
\hline & & LR (discontinued April 2000) & SR 1 & SR 2 & HR \\
\hline \multirow[t]{4}{*}{ COSS-96 } & Methotrexate & Methotrexate $^{\dagger}$ & Methotrexate & Methotrexate & Methotrexate \\
\hline & Adriamycin & Adriamycin ${ }^{\dagger}$ & Adriamycin ${ }^{\ddagger}$ & Adriamycin ${ }^{*}$ & Adriamycin \\
\hline & Cisplatin & Ifosfamide $^{\dagger}$ & Cisplatin & Cisplatin ${ }^{\ddagger}$ & Etoposide \\
\hline & Ifosfamide & & Ifosfamide & Ifosfamide & Carboplatin \\
\hline \multirow[t]{3}{*}{ Protocol } & Neoadjuvant & \multicolumn{4}{|l|}{ Adjuvant } \\
\hline & & \multicolumn{2}{|l|}{ Good responders $\|$} & \multicolumn{2}{|l|}{ Poor responders } \\
\hline & & Randomization 1 & Randomization 2 & Randomization 1 & Randomization 2 \\
\hline \multirow[t]{5}{*}{ EURAMOS-1 } & Methotrexate & Methotrexate & Methotrexate & Methotrexate & Methotrexate \\
\hline & Adriamycin & Adriamycin & Adriamycin & Adriamycin & Adriamycin \\
\hline & Cisplatin & Cisplatin & Cisplatin & Cisplatin & Cisplatin \\
\hline & & & Interferon & & Ifosfamide \\
\hline & & & & & Etoposide \\
\hline
\end{tabular}

COSS = Cooperative Osteosarcoma Study Group; EURAMOS = European and American Osteosarcoma Study Group; LR = low risk; $\mathrm{HR}=$ high risk; SR = standard risk; *intraarterial (HR 1) versus intravenous (HR 2) application; ${ }^{\dagger}$ only 9 weeks of adjuvant chemotherapy; *increased doses of adriamycin, cisplatin, and ifosfamide (SR 1) versus increased doses of methotrexate (SR 2); "less than 10\% vital tumor cells in the resected tumor. 


\section{Appendix 2: Chemotherapy for Ewing's sarcoma Protocol}

\begin{tabular}{|c|c|c|c|c|c|c|}
\hline \multirow[t]{2}{*}{ Protocol } & \multicolumn{3}{|l|}{ Neoadjuvant } & \multicolumn{3}{|l|}{ Adjuvant } \\
\hline & SR & HR 1 & HR 2 & SR & HR 1 & HR 2 \\
\hline \multirow[t]{5}{*}{ EICESS 92} & Vincristine & Vincristine & Vincristine & Vincristine & Vincristine & Vincristine \\
\hline & Actinomycin & Actinomycin & Actinomycin & Actinomycin & Actinomycin & Actinomycin \\
\hline & Ifosfamide & Ifosfamide & Ifosfamide & Ifosfamide or & Ifosfamide & Ifosfamide \\
\hline & Doxorubicin & Doxorubicin & Doxorubicin & Cyclophosphamide & Doxorubicin & Doxorubicin \\
\hline & & & Etoposide & Doxorubicin & & Etoposide \\
\hline \multirow[t]{2}{*}{ Protocol } & & \multirow[t]{2}{*}{ Neoadjuvant } & Adjuvant & & & \\
\hline & & & RG 1 & RG 2 & \multicolumn{2}{|c|}{ RG 3} \\
\hline \multirow[t]{5}{*}{ EURO-EWING 99} & & \multirow[t]{5}{*}{ VIDE } & VAI & VAI & \multicolumn{2}{|c|}{ VAI } \\
\hline & & & + VAI & + VAI & \multicolumn{2}{|c|}{+ Mel-Mel or } \\
\hline & & & or VAC & or Bu-Mel & \multicolumn{2}{|c|}{+ Tre-Mel or } \\
\hline & & & & & \multirow{2}{*}{\multicolumn{2}{|c|}{$\begin{array}{l}+ \text { Bu-Mel or } \\
+ \text { Experimental therap }\end{array}$}} \\
\hline & & & & & & \\
\hline
\end{tabular}

EICESS = European Intergroup Cooperative Ewing's Sarcoma Study; EURO-EWING = EUROpean Ewing tumour Working Initiative of National Groups;

$\mathrm{SR}=$ standard risk; $\mathrm{HR}=$ high risk; RG 1 = risk group 1 , RG $2=$ risk group 2, RG 3 = risk group 3; VIDE = vincristine, ifosfamide, doxorubicin, etoposide; VAI = vincristine, actinomycin, ifosfamide; VAC = vincristine, actinomycin, cyclophosphamide; Bu = busulfan; $\mathrm{Mel}=$ melphalan; Tre $=$ tresulfan .

\section{References}

1. Abed R, Grimer R. Surgical modalities in the treatment of bone sarcoma in children. Cancer Treat Rev. 2010;36:342-347.

2. Balamuth NJ, Womer RB. Ewing's sarcoma. Lancet Oncol. 2010;11:184-192.

3. Bath LF, Crofton PM, Evans AE, Ranke MB, Elmlinger MW, Kelnar CJ, Wallace WH. Bone turnover and growth during and after chemotherapy in children with solid tumors. Pediatr Res. 2004;55:224-230.

4. Baumgart R, Lenze U. Expandable endoprostheses in malignant bone tumors in children: indications and limitations. Recent Results Cancer Res. 2009;179:59-73.

5. Berger D, Engelbrecht R, Mertelsmann R. [The Red Book-Haematology and Internistic Oncology][in German]. Landsberg, Germany: Hüthig Jehle Rehm GmbH; 2006.

6. Bielack S, Flege S, Kempf-Bielack B. [Treatment strategies in osteosarcoma] [in German]. Der Onkologe. 2000;6:747-759.

7. Carrle D, Bielack SS. Current strategies of chemotherapy in osteosarcoma. Int Orthop. 2006;30:445-451.

8. Cool WP, Carter SR, Grimer RJ, Tillman RM, Walker PS. Growth after extendible endoprosthetic replacement of the distal femur. J Bone Joint Surg Br. 1997;79:938-942.

9. Crofton PM, Ahmed SF, Wade JC, Elmlinger MW, Ranke MB, Kelnar CJ, Wallace WH. Bone turnover and growth during and after continuing chemotherapy in children with acute lymphoblastic leukemia. Pediatr Res. 2000;48:490-496.

10. de Onis M. Update on the implementation of the WHO child growth standards. World Rev Nutr Diet. 2013;106:75-82.

11. de Onis M, Garza C, Victora CG, Onyango AW, Frongillo EA, Martines J. The WHO Multicentre Growth Reference Study: planning, study design, and methodology. Food Nutr Bull. 2004;25(1 suppl):S15-26.

12. Ecklund K, Laor T, Goorin AM, Connolly LP, Jaramillo D. Methotrexate osteopathy in patients with osteosarcoma. Radiology. 1997;202:543-547.

13. Fan C, Foster BK, Wallace WH, Xian CJ. Pathobiology and prevention of cancer chemotherapy-induced bone growth arrest, bone loss, and osteonecrosis. Curr Mol Med. 2011;11:140-151.

14. Glasser DB, Duane K, Lane JM, Healey JH, Caparros-Sison B. The effect of chemotherapy on growth in the skeletally immature individual. Clin Orthop Relat Res. 1991;262:93-100.

15. Greulich WW, Pyle SI. Radiographic Atlas of Skeletal Development of the Hand and Wrist. 2nd ed. Stanford, CA: Stanford University Press; 1959.

16. Groot-Loonen JJ, Otten BJ, van t'Hof MA, Lippens RJ, Stoelinga GB. Chemotherapy plays a major role in the inhibition of catchup growth during maintenance therapy for childhood acute lymphoblastic leukemia. Pediatrics. 1995;96:693-695.

17. Hokken-Koelega AC, van Doorn JW, Hahlen K, Stijnen T, de Muinck Keizer-Schrama SM, Drop SL. Long-term effects of treatment for acute lymphoblastic leukemia with and without cranial irradiation on growth and puberty: a comparative study. Pediatr Res. 1993;33:577-582.

18. Hwang N, Grimer RJ, Carter SR, Tillman RM, Abudu A, Jeys LM. Early results of a non-invasive extendible prosthesis for limb-salvage surgery in children with bone tumours. J Bone Joint Surg Br. 2012;94:265-269.

19. Juergens C, Weston C, Lewis I, Whelan J, Paulussen M, Oberlin O, Michon J, Zoubek A, Juergens H, Craft A. Safety assessment of intensive induction with vincristine, ifosfamide, doxorubicin, and etoposide (VIDE) in the treatment of Ewing tumors in the 
EURO-E.W.I.N.G. 99 clinical trial. Pediatr Blood Cancer. 2006;47:22-29.

20. Kotz RI, Windhager R, Dominkus M, Robioneck B, MullerDaniels H. A self-extending paediatric leg implant. Nature. 2000;406:143-144.

21. Ladenstein R, Potschger U, Le Deley MC, Whelan J, Paulussen M, Oberlin O, van den Berg H, Dirksen U, Hjorth L, Michon J, Lewis I, Craft A, Jurgens H. Primary disseminated multifocal Ewing sarcoma: results of the Euro-EWING 99 trial. J Clin Oncol. 2010;28:3284-3291.

22. Lewis M. The use of an expandable and adjustable prosthesis in the treatment of childhood malignant bone tumors of the extremity. Cancer. 1986;57:499-502.

23. Paley D, Bhave A, Herzenberg JE, Bowen JR. Multiplier method for predicting limb-length discrepancy. J Bone Joint Surg Am. 2000;82:1432-1446.

24. Paley J, Talor J, Levin A, Bhave A, Paley D, Herzenberg JE. The multiplier method for prediction of adult height. $J$ Pediatr Orthop. 2004;24:732-737.

25. Parkes SE, Parke S, Mangham DC, Grimer RJ, Davies $P$, Morland BJ. Fifty years of paediatric malignant bone tumours in the West Midlands, UK, 1957-2006: incidence, treatment and outcome. Paediatr Perinat Epidemiol. 2010;24:470-478.

26. Paulussen M, Craft AW, Lewis I, Hackshaw A, Douglas C, Dunst J, Schuck A, Winkelmann W, Kohler G, Poremba C, Zoubek A, Ladenstein R, van den Berg H, Hunold A, Cassoni A, Spooner D, Grimer R, Whelan J, McTiernan A, Jurgens H, European Intergroup Cooperative Ewing's Sarcoma Study-92. Results of the EICESS-92 study: two randomized trials of Ewing's sarcoma treatment: cyclophosphamide compared with ifosfamide in standard-risk patients and assessment of benefit of etoposide added to standard treatment in high-risk patients. $J$ Clin Oncol. 2008;26:4385-4393.

27. Pirker-Fruhauf UM, Friesenbichler J, Urban EC, ObermayerPietsch B, Leithner A. Osteoporosis in children and young adults: a late effect after chemotherapy for bone sarcoma. Clin Orthop Relat Res. 2012;470:2874-2885.

28. Ritter J, Bielack SS. Osteosarcoma. Ann Oncol. 2010;21(suppl 7):vii320-325.

29. Robson H. Bone growth mechanisms and the effects of cytotoxic drugs. Arch Dis Child. 1999;81:360-364.

30. Robson H, Anderson E, Eden OB, Isaksson O, Shalet S. Chemotherapeutic agents used in the treatment of childhood malignancies have direct effects on growth plate chondrocyte proliferation. J Endocrinol. 1998;157:225-235.
31. Roche AF, Wainer H, Thissen D. The RWT method for the prediction of adult stature. Pediatrics. 1975;56:1027-1033.

32. Sanders JO, Howell J, Qiu X. Comparison of the Paley method using chronological age with use of skeletal maturity for predicting mature limb length in children. J Bone Joint Surg Am. 2011;93:1051-1056.

33. Schiller C, Windhager R, Fellinger EJ, Salzer-Kuntschik M, Kaider A, Kotz R. Extendable tumour endoprostheses for the leg in children. J Bone Joint Surg Br. 1995;77:608-614.

34. Schleiermacher G, Peter M, Oberlin O, Philip T, Rubie H, Mechinaud F, Sommelet-Olive D, Landman-Parker J, Bours D, Michon J, Delattre O; Société Française d'Oncologie Pédiatrique. Increased risk of systemic relapses associated with bone marrow micrometastasis and circulating tumor cells in localized Ewing tumor. J Clin Oncol. 2003;21:85-91.

35. Tanner JM, Healy MJR, Goldstein H, Cameron N. Assessment of Skeletal Maturity and Prediction of Adult Height (TW3) Method. 3rd ed. London, UK: WB Saunders; 2001.

36. van Leeuwen BL, Hartel RM, Jansen HW, Kamps WA, Hoekstra HJ. The effect of chemotherapy on the morphology of the growth plate and metaphysis of the growing skeleton. Eur J Surg Oncol. 2003;29:49-58.

37. van Leeuwen BL, Kamps WA, Hartel RM, Veth RP, Sluiter WJ, Hoekstra HJ. Effect of single chemotherapeutic agents on the growing skeleton of the rat. Ann Oncol. 2000;11:1121-1126.

38. van Leeuwen BL, Kamps WA, Jansen HW, Hoekstra HJ. The effect of chemotherapy on the growing skeleton. Cancer Treat Rev. 2000;26:363-376.

39. Viana MB, Vilela MI. Height deficit during and many years after treatment for acute lymphoblastic leukemia in children: a review. Pediatr Blood Cancer. 2008;50(2 suppl):509-516; discussion 517.

40. Winkler K, Bielack S, Delling G, Salzer-Kuntschik M, Kotz R, Greenshaw C, Jurgens H, Ritter J, Kusnierz-Glaz C, Erttmann R, et al. Effect of intraarterial versus intravenous cisplatin in addition to systemic doxorubicin, high-dose methotrexate, and ifosfamide on histologic tumor response in osteosarcoma (study COSS-86). Cancer. 1990;66:1703-1710.

41. Xian CJ, Cool JC, Scherer MA, Macsai CE, Fan C, Covino M, Foster BK. Cellular mechanisms for methotrexate chemotherapyinduced bone growth defects. Bone. 2007;41:842-850.

42. Xian CJ, Cool JC, van Gangelen J, Foster BK, Howarth GS. Effects of etoposide and cyclophosphamide acute chemotherapy on growth plate and metaphyseal bone in rats. Cancer Biol Ther. 2007;6:170-177. 